\title{
Reabilitação bucal com prótese obturadora maxilar após excisão de carcinoma adenoide cístico
}

Mouth rehabilitation with maxillary obturator prosthesis after excision of adenoid cystic carcinoma Rehabilitación bucal con prótesis obturadora maxilar tras excision de carcinoma adenoide quístico

\section{Elizabeth Gomes DA COSTA ${ }^{1}$} Bruno Maia DE LIMA²

Rayane Torres DA MATA2

Sybilla Torres DIAS ${ }^{3}$

Brigitte NICHTHAUSER

Cristiane Maria Brasil LEAL ${ }^{3}$

'Graduanda em Odontologia pela Universidade do Estado do Amazonas (UEA), 69065-001 Manaus - AM, Brasil

${ }^{2}$ Cirurgiã(o) Dentista, 69065-001 Manaus - AM, Brasil

${ }^{3}$ Departamento de Prótese Total e Bucomaxilofacial da Universidade do Estado do Amazonas (UEA), 69065-001 Manaus - AM, Brasil

\section{Resumo}

A prótese obturadora constitui o principal método para a reabilitação de grandes defeitos maxilares. Tem como objetivo fechar o defeito, separar a cavidade oral da cavidade nasal, prevenir a fala anasalada e a regurgitação nasal de alimentos e líquidos e dar sustentação ao perfil facial. Este artigo visa relatar um caso de reabilitação com prótese obturadora maxilar após maxilectomia parcial. Paciente do gênero feminino, 72 anos, diagnosticada com carcinoma adenoide cístico na região de palato, foi submetida a maxilectomia parcial que resultou em extenso defeito maxilar e comunicação bucosinusal. Após anamnese, exames extra, intraorais e radiográfico, foi planejado para o período cicatricial e de uso da sonda nasogástrica, a confecção de uma placa obturadora para melhorar a deglutição e fala. Em seguida, confecção de uma prótese total obturadora superior e prótese parcial removível inferior. A placa obturadora foi confeccionada e utilizada até a remoção da sonda e instalação das próteses. Após moldagem das duas arcadas, foram obtidos modelos funcionais que foram montados em articulador após os ajustes dos planos de orientação e registro das relações intermaxilares. Foram realizadas provas estéticas e funcionais dos dentes artificiais e ajustes necessários. Posteriormente à acrilização, as próteses foram instaladas e 0 ajuste oclusal executado. Para obter retenção e estabilidade satisfatórias durante a utilização da prótese obturadora superior foi sugerido o uso de adesivo para dentadura. Três proservações foram realizadas. A prótese obturadora reabilitou a estética, fonética, função mastigatória e deglutição restabelecendo a autoestima e a ressocialização da paciente.

Descritores: Obturadores Palatinos; Prótese Maxilofacial; Reabilitação Bucal; Carcinoma Adenoide Cístico.

\section{Abstract}

The obturator prosthesis is the main method for the rehabilitation of large maxillary defects. With the purpose to close the defect, separate the oral cavity from the nasal cavity, prevent hypernasal speech, nasal regurgitation of food and liquids and provide support for the facial profile. This article aims to report a case of rehabilitation with maxillary obturator prosthesis after partial maxillectomy. A 72-year-old female patient, diagnosed with adenoid cystic carcinoma in the palate region, underwent partial maxillectomy that resulted in extensive maxillary defect and bucco-sinus communication. After anamnesis, extra, intraoral and radiographic exams, the construction of a palatal plate was planned to be used with the nasogastric tube and during the healing process, to improve swallowing and speech. Then, the manufacture of a maxillary obturator prosthesis and a lower removable partial prosthesis. The palatal plate was made and used until the tube was removed and the insertion of the prostheses. After the impressions, master casts were made and mounted on an articulator after adjustment of wax occlusion rims and registration of the maxillo-mandibular relations. Functional elements of speech, occlusion, and appearance were evaluated during the try-in of the provisional set-up and adjusts were made. After the acrylization, the prostheses were inserted and the occlusal adjustment performed. To obtain satisfactory retention and stability when using the maxillary obturator prosthesis, it was suggested to use denture adhesive. Three post-insertions visits were carried out. The obturator prosthesis rehabilitated aesthetics, phonetics, masticatory function and swallowing, restoring the patient's self-esteem and improving social life.

Descriptors: Palatal Obturators; Maxillofacial Prosthesis; Mouth Rehabilitation; Adenoid Cystic Carcinoma.

\section{Resumen}

La prótesis obturadora es el método principal para la rehabilitación de grandes defectos maxilares. Tiene por objetivo cerrar el defecto, separar la cavidad bucal de la cavidad nasal, prevenir el habla nasal, la regurgitación nasal de alimentos y líquidos, dando apoyo al perfil facial. Este artículo tiene objetivo de reportar un caso de rehabilitación con prótesis obturadora maxilar tras maxilectomía parcial. Una paciente de 72 años, diagnosticada de carcinoma adenoide quístico en región del palato, fué sometida a maxilectomía parcial que resultó en extenso defecto maxilar y comunicación bucosinusal. Después de anamnesis, exámenes extra, intraorales y radiográficos, se planificó para el período de cicatrización y el uso de la sonda nasogástrica, la construcción de una placa obturadora para mejorar la deglución y el habla. Luego, la fabricación de una prótesis obturadora total superior y una prótesis parcial inferior removible. La placa obturadora se hizo y se utilizó hasta que se retiró la sonda y se instalaron las prótesis. Se realizón la impresión de ambos arcos, obtuvieron modelos funcionales que se montaron en articulador, tras ajustes de los planos de orientación y registro de relaciones intermaxilares. Se realizaron pruebas estéticas y funcionales en dientes artificiales y ajustes necesarios. Después de la acrilización se instalaron las prótesis y se realizó ajuste oclusal. Para obtener una retención y estabilidad satisfactoria al utilizar la prótesis obturadora superior, fue sugirió utilizar un adesivo para prótesis. Se realizaron tres proservaciones. La prótesis obturatriz rehabilitó estética, fonética, función masticatoria y deglución, devolviendo autoestima y resocialización al paciente.

Descriptores: Obturadores Palatinos; Prótesis Maxilofacial; Rehabilitación Bucal; Carcinoma Adenoide Quístico.

INTRODUÇÃO

O Carcinoma Adenóide Cístico (CAC) é uma neoplasia maligna de glândulas salivares que afeta predominantemente as glândulas salivares menores, tendo o palato como sítio oral mais habitual ${ }^{1}$. Apresenta-se clinicamente como um aumento de volume de crescimento lento, porém de curso invasivo e indolor, que acomete principalmente adultos de meia idade e apresenta, de acordo com alguns estudos, discreta predileção pelo sexo feminino ${ }^{2,3}$. O 
tratamento de escolha do CAC é a excisão cirúrgica completa da lesão, com margem de segurança, devido sua característica infiltrativa responsável pela incidência de recidivas e metástases $^{2,4}$ Nas lesões que acometem o palato duro, a maxilectomia parcial ou total é o tratamento de escolha, seguida de quimioterapia e/ou radioterapia ${ }^{2,4}$.

A maxilectomia parcial ou total consiste na remoção de uma parte ou de todo o osso maxilar. Apesar de atender às necessidades do tratamento do CAC, esta técnica provoca uma comunicação bucosinusal que prejudica a estética da cavidade bucal, acarreta deficiência da função mastigatória, da capacidade de deglutição e da fonética, mostrando-se, portanto, necessária a correção do defeito causado a fim de oferecer qualidade de vida ao paciente ${ }^{5,6}$.

As abordagens terapêuticas para pacientes submetidos a maxilectomia envolvem enxertos não vascularizados, transferência de tecido livre, retalhos locais e regionais e obturadores protéticos ${ }^{7}$. No entanto, a reabilitação por meio de uma prótese obturadora é comumente a intervenção mais adotada em comparação com os meios cirúrgicos, devido à vantagem de não ser um procedimento invasivo e por permitir, através de sua possibilidade de remoção, a descoberta precoce de eventual recidiva da lesão ao exame clínico ${ }^{8}$.

A prótese obturadora maxilar é um dispositivo que consiste em substituir a área removida, obliterando a comunicação bucosinusal existente e reparando o defeito maxilar, podendo estar associada à armação de prótese parcial removível ou à base de prótese total $^{8-10}$.

Existem dois tipos de próteses obturadoras, as próteses palatinas e próteses velo-palatinas. As próteses obturadoras palatinas são as mais utilizadas na ressecção do palato duro e maxila, constituídas por uma parte superior, adaptada na cavidade obturadora e uma parte inferior que permite o restabelecimento do contorno da abóbada palatina. Além disto, as próteses obturadoras são classificadas em próteses cirúrgicas ou imediatas que são planejadas antes do procedimento cirúrgico, próteses provisórias, frequentemente usadas durante o processo de cicatrização e as próteses definitivas que são posicionadas apenas após a estabilização dos tecidos orais adjacentes ${ }^{11,12}$.

Após a confecção e instalação de uma prótese obturadora maxilar, as funções bucais são restabelecidas e a face adquire suporte novamente, tornando possível a reabilitação funcional e estética do paciente bem como a devolução de sua autoestima e retorno ao convívio social $^{9,12}$.

Diante disto, é de grande relevância o conhecimento teórico e prático a respeito desta abordagem clínica, visto que a prótese obturadora reabilita o paciente em relação à função e estética, além de possibilitar a proservação da lesão ou eventual recidiva por meio do exame clínico com remoção da prótese. Sendo assim, este artigo tem como objetivo relatar um caso clínico de paciente oncológica reabilitada com prótese obturadora maxilar após maxilectomia parcial para excisão de CAC.

CASO CLÍNICO

Paciente do gênero feminino, 72 anos, leucoderma, procurou o Centro de Especialidades Odontológica da Universidade do Estado do Amazonas queixando-se da necessidade de reabilitação bucal. $\mathrm{Na}$ anamnese, relatou ter sido submetida a cirurgia para remoção de um carcinoma adenoide cístico na região do palato, que resultou em extenso defeito maxilar e comunicação bucosinusal. Por isto, apresentava problemas funcionais na fonação, mastigação e deglutição além de insatisfação estética. Relatou também que gozava de boa saúde e não apresentava nenhum problema sistêmico que pudesse comprometer o tratamento. Ao exame clínico extraoral verificou-se ausência de dor a palpação e inexistência de tumefação (Figura 1).

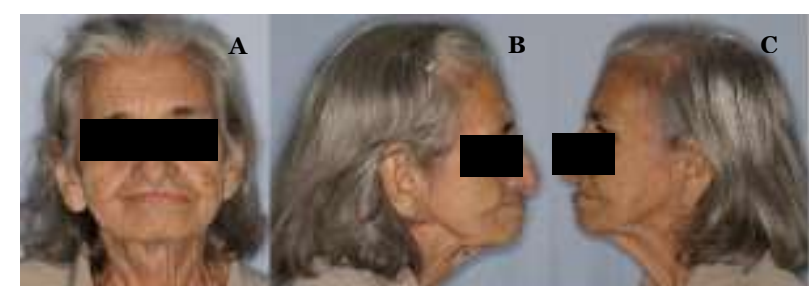

Figura 1: Aspecto extraoral inicial. A) Frontal. B) Perfil direito. C) Perfil esquerdo.

Ao exame clínico intraoral foi constatado comunicação bucosinusal na hemiarcada superior direita, ausência de elementos dentários na hemiarcada superior esquerda e presença dos dentes 33 a 42 na arcada inferior que apresentavam desgastes incisais, ausência de cárie e de lesão periodontal (Figura 2). Após análise dos exames, considerando que a paciente se recusava a qualquer intervenção cirúrgica reparadora e que ainda se encontrava em fase de cicatrização da cirurgia, foi planejada a confecção de uma placa obturadora em resina acrílica com a finalidade de melhorar a deglutição e a fonética e, em seguida, confecção de prótese total obturadora superior e prótese parcial removível (PPR) inferior. 


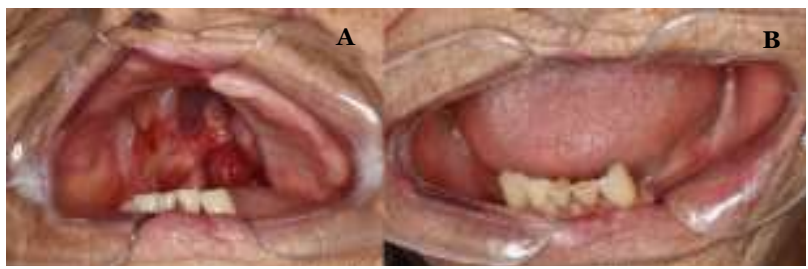

Figura 2: Aspecto inicial da cavidade bucal. A) Arcada superior. B) Arcada inferior.

Após moldagem da arcada superior com hidrocoloide irreversível, foi obtido o modelo de gesso no qual foi encerada e polimerizada em resina acrílica incolor a placa obturadora palatina. A placa obturadora foi ajustada e instalada. A paciente relatou boa estabilidade e retenção além de melhora significativa na deglutição e fonética (Figura 3).

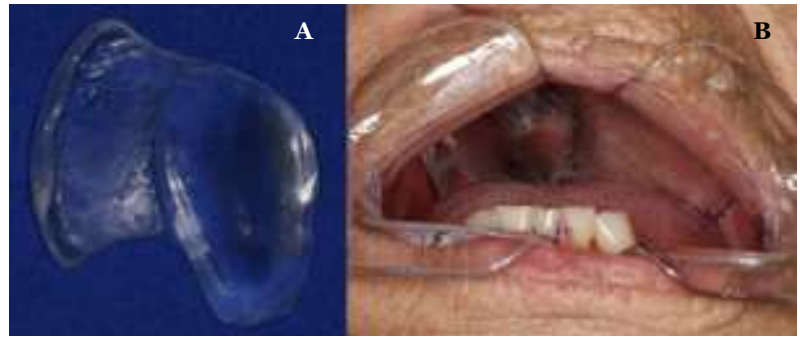

Figura 3: A) Placa obturadora. B) Placa obturadora instalada.

Em seguida, as arcadas superior $e$ inferior foram moldadas com hidrocoloide irreversível (Figura 4), sendo a superior moldada com material em consistência mais espessa reproduzindo a extensão e profundidade da comunicação bucosinusal e, foram obtidos os modelos de gesso superior e inferior (Figura 5). No modelo inferior foi realizado o delineamento para determinação do eixo de inserção da PPR, confecção das coroas guias e desenho da armação metálica com 0 grampo contínuo de Kennedy.

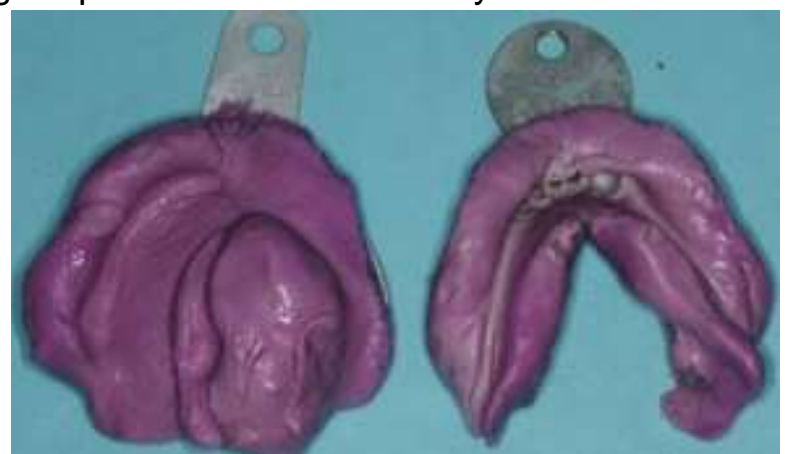

Figura 4: Moldes das arcadas superior e inferior

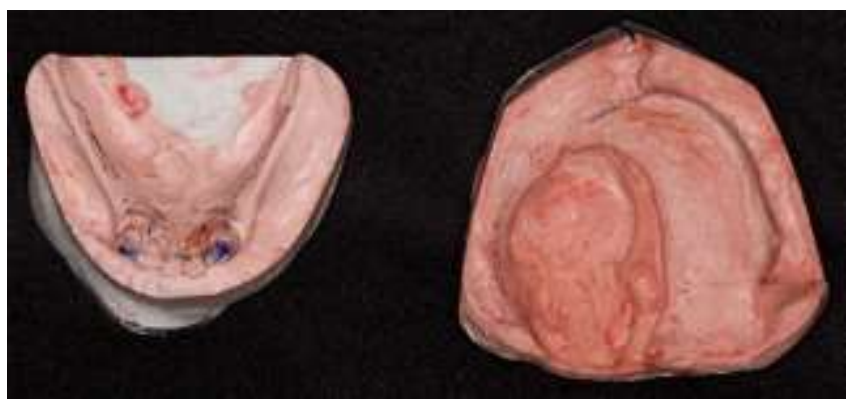

Figura 5: Modelos de gesso das arcadas inferior e superior.
Após adequação do meio bucal com a confecção dos nichos e desgastes compensatórios nos dentes inferiores, foi realizada a moldagem com silicone de condensação e obtido o modelo de trabalho inferior sobre o qual foi confeccionada a armação metálica da PPR. Sobre o modelo superior foi confeccionado o plano de cera da prótese total obturadora que posteriormente foi ajustado na boca da paciente seguindo seu perfil facial e oclusal. A armação metálica foi provada em boca. Após registro do arco facial, o modelo superior foi montado em articulador semiajustável e, em seguida, foram determinadas as relações intermaxilares, relação cêntrica e dimensão vertical de oclusão, para montagem do modelo inferior. Os dentes artificiais foram selecionados tomando como referência os dentes remanescentes da paciente, as linhas de referência demarcadas no plano de cera e características da paciente como sexo, formato do rosto e idade. Em seguida, foi realizada a montagem dos dentes artificiais (Figuras 6 e 7 ).

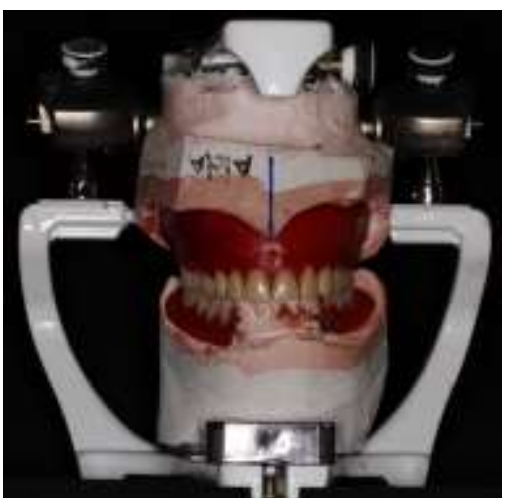

Figura 6: Dentes artificiais montados em cera.

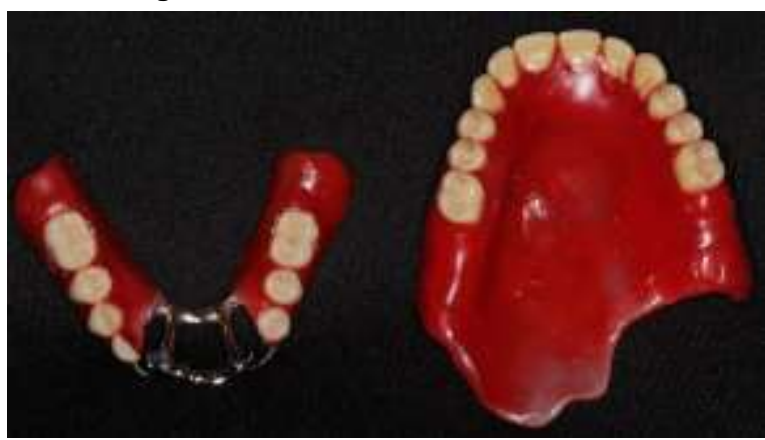

Figura 7: Próteses com os dentes montados

Foi realizada a prova estética e funcional dos dentes artificiais na cavidade bucal e avaliados o suporte de lábio, altura incisal, corredor bucal, características dos dentes artificiais como cor, tamanho e formato, linha mediana, linha alta do sorriso, testes fonéticos, testes de retenção e estabilidade além de ajustes oclusais (Figura 8). Após ter sido constatado o restabelecimento estético, fonético e oclusal da paciente, foi realizada a moldagem funcional com boca fechada utilizando-se 
silicone de condensação de consistência leve (Figura 9).

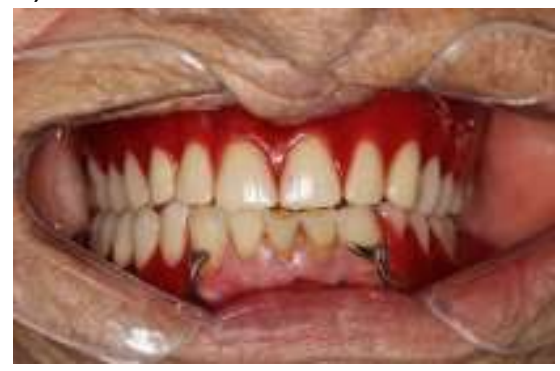

Figura 8: Prova estética e funcional dos dentes artificiais.

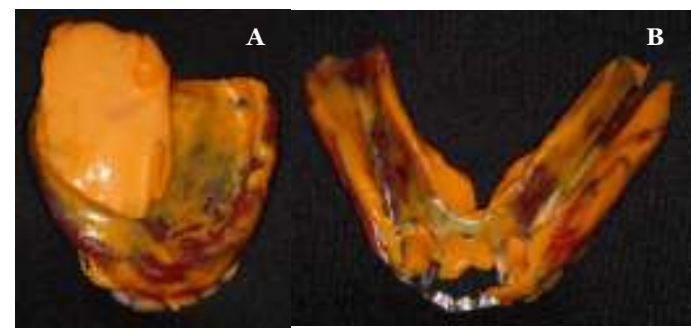

Figura 9: Bases das próteses após moldagem com boca fechada. A) Superior. B) Inferior.

As próteses seguiram para as fases laboratoriais de acrilização, acabamento e polimento (Figura 10).

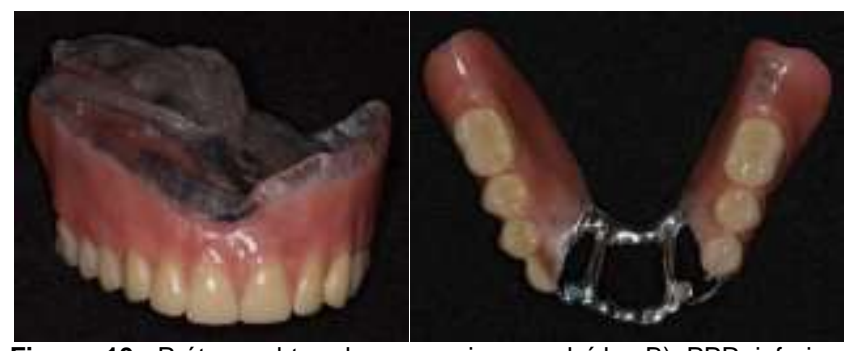

Figura 10: Prótese obturadora superior concluída. B) PPR inferior concluída.

As próteses foram instaladas e a paciente recebeu, por escrito, informações sobre higienização e cuidados necessários para a manutenção das mesmas (Figuras 11 e 12).

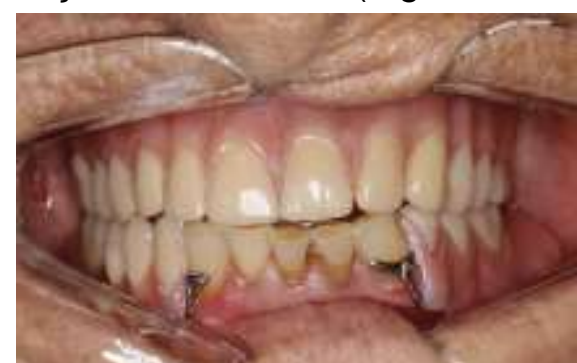

Figura 11: Próteses instaladas em oclusão.

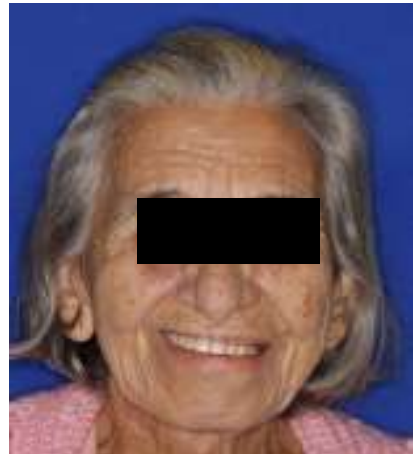

Figura 12: Sorriso após instalação das próteses.
$\mathrm{Na}$ primeira proservação, realizada 24 horas após instalação, foram executados pequenos ajustes e a paciente relatou satisfação com a estética, com a fala que se tornou compreensível e melhora significativa na mastigação e deglutição, além do restabelecimento da autoestima e retorno ao convívio social. Foi sugerido a paciente que para melhorar ainda mais a retenção da prótese, poderia ser utilizado adesivo para dentadura. Em seguida, foram realizadas proservações nos períodos de 48 horas e uma semana aonde foi constatado que a prótese ficou bem adaptada. A paciente recebeu acompanhamento no $15^{\circ}$ e $30^{\circ}$ dia após a instalação da prótese e em seguida foi orientada a retornar após três meses.

DISCUSSÃO

As neoplasias malignas que afetam as glândulas salivares são relativamente raras, representando menos de $7 \%$ dos cânceres de cabeça e pescoço. Destes, apenas $10 \%$ são diagnosticados como $\mathrm{CAC}^{2,4}$. O CAC na cavidade bucal surge comumente das glândulas salivares menores do palato duro; geralmente acomete pacientes após a quarta década de vida, com predileção pelo gênero feminino ${ }^{1,2}$. Clinicamente apresenta um aumento de volume lento, porém de curso invasivo e em estágios mais avançados, pode ocorrer destruição óssea e invasão perineural causando dor e perda da função do nervo facial ou paralisia ${ }^{3,4}$. Seu tratamento é a remoção completa da lesão que, quando localizada no palato duro, tem como tratamento indicado a maxilectomia parcial ou total $^{2}$. No caso relatado neste trabalho, a paciente é do gênero feminino, sétima década de vida, foi diagnosticada com CAC e submetida a maxilectomia parcial.

As maxilectomias parciais ou totais podem provocar comunicação bucosinusal gerando problemas na deglutição, mastigação, fala, estética, aceitação psicológica e socialização ${ }^{7,13}$. Conforme Moss et al. ${ }^{7}$, Chen et al. ${ }^{13}$ e Rodrigues et al. ${ }^{14}$ a prótese obturadora constitui o principal método para a reabilitação de grandes defeitos maxilares tendo como objetivo, fechar a comunicação bucosinusal separando a cavidade oral das nasais e assim, prevenindo a fala anasalada, a regurgitação nasal de alimentos e líquidos e dando sustentação ao perfil facial. O caso clínico corroborou com a opinião destes autores tendo sido confeccionada inicialmente uma placa obturadora e em seguida uma prótese total obturadora, sendo que ambas auxiliaram na reabilitação da paciente. 
O estudo de Tirelli et al. ${ }^{14}$ salienta a importância de cirurgia plástica reconstrutiva ou prótese obturadora como tratamentos de maxilectomias, afirmando porém, que a prótese obturadora oferece vantagens como possibilidade de restaurar imediatamente a estética e a função sem precisão de novas cirurgias e permitir manter a cavidade sob controle em caso de recidiva da neoplasia. O caso clínico foi conduzido observando as vantagens relatas por estes autores visto que 0 CAC possui altas prevalências de recorrências.

Para Miyashita et al. ${ }^{11}$ e Kusterer et al. ${ }^{16}$, é importante no período pós-operatório uma prótese ou placa obturadora de palato provisória para proporcionar ao paciente alimentação por via oral, deglutição e fala. Auxiliando assim, na cicatrização e evitando contaminação na ferida enquanto se realiza o planejamento da prótese obturadora maxilar. No caso clínico descrito, a paciente procurou atendimento odontológico logo após realização da maxilectomia parcial, na fase de cicatrização cirúrgica e com a sonda nasogátrica, por isto, inicialmente foi confeccionada uma placa obturadora em resina acrílica autopolimerizável e em seguida a prótese total obturadora maxilar e prótese total convencional inferior.

Segundo Singh et al. ${ }^{17}$ a ausência de retenção, estabilidade e suporte é o problema mais comum com tratamento protético de pacientes com maxilectomia parcial. Por isto, será comum ao instalar obturador protético maxilar, o grau de movimento variar com o número e posição dos dentes remanescentes, a dimensão e configuração do defeito, quantidade e contorno da área palatal remanescente, altura da crista alveolar remanescente e a dimensão, contorno e mucosa do revestimento do defeito. Os pacientes que apresentam quantidade adequada de dentes e estruturas de suporte adjacentes apresentam uma melhor retenção e estabilidade da prótese ${ }^{16}$. O efeito do peso intrínseco da prótese obturadora maxilar sobre os tecidos de suporte representa um fator agravante, sendo fundamental a adequada avaliação dos tecidos remanescentes para o bom desempenho da prótese e preservação das estruturas anatômicas de retenção e suporte ${ }^{11}$. Graziani $^{18}$ sugeriu que uma prótese volumosa, destinada a obturar uma ampla cavidade, tivesse a parte superior intracavitária oca, o que denominou de "prótese oca". No caso relato, a paciente era edentada total e tinha uma ampla cavidade, no entanto, a parte superior da prótese que adaptava na cavidade obturador foi densa, a retenção da prótese foi obtida sobre o rebordo residual, tecido mucoso remanescente e zona retentiva que a cavidade apresentava. Foi sugerido a paciente o uso de adesivo para dentadura caso necessitasse de maior retenção.

Os hidrocoloides irreversíveis nos permitem realizar moldagens preliminares cuidadosas no paciente, pois as suas propriedades químicas e físicas possibilitam fácil manipulação, boa fluidez, tempo de trabalho ajustável e boa adaptação aos tecidos bucais, preservando e evitando iatrogenias ao paciente ${ }^{6}$. Singh et al. $^{17}$ preconizam a manipulação de hidrocoloides irreversíveis para moldagem em paciente com hemimaxilectomia e cicatrização completa do local operado, para obtenção de prótese obturadora. Fundamentados na literatura, foi realizada a manipulação e utilização de hidrocoloides irreversíveis como material de moldagem, evitando desconfortos e iatrogênicas a paciente. No entanto, após a prova estética e funcional dos dentes artificiais, foi realizada a moldagem com boca fechada, utilizando-se silicone de condensação.

A proservação é fator importante para se obter melhores resultados. Neste caso, após a instalação da prótese obturadora e prótese parcial removível, a paciente foi orientada a retornar em 24 horas, a fim de avaliar a adaptação, presença de feridas ou aftas, além da avaliação da retenção e estabilidade da prótese. Quanto à retenção, a título de melhora, foi orientada a fazer uso de adesivos para dentaduras. Quanto a feridas, não estavam presentes, sendo necessário apenas alguns pequenos ajustes, além de não haver sensação de dor. Ao contrário, no caso relatado por Rabelo et al. ${ }^{6}$, a paciente queixou-se de dores na região limítrofe, entre palato duro e mole.

CONCLUSÃO

A placa obturadora permite a diminuição do tempo de uso da sonda nasogástrica, veda a comunicação bucosinusal permitindo que o paciente retorne a dieta por via oral, melhora a fonética e contribui para a recuperação psicológica. A prótese obturadora maxilar reabilita o paciente submetido a maxilectomia parcial restabelecendo a estética, fonética, função mastigatória e deglutição devolvendo a autoestima e a ressocialização.

\section{REFERÊNCIAS}

1. Neville BW, Damm DD, Allen CM, Bouquot JE. Patologia oral e maxilofacial. Rio de Janeiro: Elsevier; 2016.

2. Kumar AN, Harish M, Alavi YA, Mallikarjuna R. Adenoid cystic carcinoma of buccal mucosa. BMJ Case Rep. 2013;2013:bcr2013009770.

3. De Brito Monteiro BV, Grempel RG, Da Costa 
Miguel MC, Adenoidcystic Carcinoma Mimickingan Oroantral Fístula: A Case Report. International Arch Otorhinolaryngol. 2014;18(2): 221-25.

4. Severo MLB, Barros CCS, Rocha LCON, Macêdo LS, Miguel MCCM, Silveira EJD. Carcinoma adenoide cístico: imunohistoquímica e diagnóstico diferencial, um relato de caso. J Bras Pantol Med Lab. 2019;55(5):488-97.

5. Aguiar L, Mozzini AR, Lersch E, De Conto F. Obturador palatino: confecção de uma prótese não convencional - relato de caso. RFO. 2013; 18(1):125-9.

6. Rabelo DP, Tanure RP, Grajeda FM, Grossmann SM. Reabilitação Protética Oral em Paciente Oncológico. rEV UninCor. 2018;16(1): 1-8.

7. Moss OB, Pinheiro BCL, Mendes TCC, Braga FP, Nichthauser B, Leal CMB. Arch Health Invest. 2019;8(11):706-11.

8. Goiato MC, Pesqueira AA, Ramos da Silva $C$, Gennari Filho H, Dos Santos M. Patient satisfaction with maxillofacial prosthesis. Literature review. J Plast Reconstr Aesthet Surg. 2009;62(2):175-80.

9. Hoshiai $T$, lida $T$, Taniguchi $H$. Vibratory properties of maxillary dentition in maxillectomy patients wearing metal framework obturator prostheses with three different metal materials. J Prosthodont Res. 2011;55(4):252-61.

10. Meenakshi A, Shah D. The obturador prostheses for maxillectomy, SRM J Res Dent Sci. 2012;3(3):193-97.

11. Miyashita E, Mattos B, Marafon P. Sistemas de retenção em prótese obturadora cirúrgica para pacientes maxilectomizados. Pesq Bras Odontoped Clín Integr. 2011;11(2):263-68.

12. Depprich R, Naujoks C, Lind D, Ommerborn M, Meyer U, Kübler NR, Handschel J. Evaluation of the quality of life of patients with maxillofacial defects after prosthodontic therapy with obturator prostheses. Int J Oral Maxillofac Surg. 2011;40:71-9.

13. Chen C, Ren W, Gao L, Cheng Z, Zhang L, Li $S$, et al. Function of obturator prosthesis after maxillectomy and prosthetic obturator rehabilitation. Braz J Otorhinolaryngol. 2016; 82(2):177-83.

14. Rodrigues RG, Rodrigues DS, Olivieira DC. Reabilitação com Prótese Bucomaxilofacial: Revisão de Literatura. RSM. 2019;5:20-7.

15. Tirelli $G$, Biasotto $M$, Lenarda RD, Argenti $B$, Gatto A, Bullo F. Obturator prostheses following palatal resection: clinical case. Acta Otrhinolaryngol Italica. 2010;30:33-9.

16. Kusterer LEFL, Paraguassú GM, Silva VSM, Sarmento VA. Reabilitação com obturador maxilar após cirurgia oncológica: relato de casos. Rev Cir Traumatol Bucomaxilofac. 2012;12 (4):9-16.

17. Singh $M$, Bhushan $A$, Kumar $N$, Chand $S$. Obturator prosthesis for hemimaxillectomy patients. Nat J Maxillofac Surg. 2013;4(1): 117-20.

18.Graziani M. Prótese Maxilo-faccial. Rio de Janeiro: Guanabara Koogan; 1982.

\section{CONFLITO DE INTERESSES}

Os autores declaram não haver conflitos de interesse

\section{AUTOR PARA CORRESPONDÊNCIA}

\section{Cristiane Maria Brasil Leal}

Avenida: Coronel Teixeira, 2104, quadra B, casa 9 Condomínio Ponta Negra Village, Ponta Negra 69030-480 Manaus - AM, Brasil

E-mail: cbleal@uea.edu.br
Submetido em 05/11/2020 Aceito em 16/07/2021 